\title{
BMI open Screening for congenital cytomegalovirus infection using newborn urine samples collected on filter paper: feasibility and outcomes from a multicentre study
}

\author{
Shin Koyano, ${ }^{1}$ Naoki Inoue, ${ }^{2}$ Akira Oka, ${ }^{3}$ Hiroyuki Moriuchi, ${ }^{4}$ Kimisato Asano, ${ }^{5}$ \\ Yushi Ito, ${ }^{6}$ Hideto Yamada, ${ }^{7}$ Tetsushi Yoshikawa, ${ }^{8}$ Tatsuo Suzutani, ${ }^{9}$ for the \\ Japanese Congenital Cytomegalovirus Study Group
}

To cite: Koyano S, Inoue N, Oka A, et al. Screening for congenital cytomegalovirus infection using newborn urine samples collected on filter paper: feasibility and outcomes from a multicentre study. BMJ Open 2011;1:000118. doi:10.1136/ bmjopen-2011-000118

- Prepublication history and additional figures for this paper are available online. To view these files please visit the journal online (http:// bmjopen.bmj.com).

Received 10 March 2011 Accepted 24 June 2011

This study was presented in part at the Congenital Cytomegalovirus Workshop, 22-24 September 2010, Paris, France.

This final article is available for use under the terms of the Creative Commons Attribution Non-Commercial 2.0 Licence; see http://bmjopen.bmj.com

For numbered affiliations see end of article.

Correspondence to Dr Naoki Inoue; ninoue@nih.go.jp

\section{ABSTRACT}

Background: As congenital cytomegalovirus (CMV) infection causes significant clinical consequences not only at birth but also later as neurological sequelae, it is critical to establish a strategy for screening congenitally infected newborns. Previous studies have identified an insufficient sensitivity in screening methods based on the use of dried blood spots (DBSs).

Objectives: To evaluate the feasibility of the authors' recently developed method for large-scale screening for congenital CMV infection and to identify risk factors for congenital infection.

Methods: More than 21000 newborns were enrolled at 25 sites in six geographically separate areas of Japan. Urine was collected onto filter cards placed in the diapers, which were then analysed by quantitative PCR using the filter disc directly as a template. Clinical and physical findings of the newborns were extracted from their medical records. CMV strains from the cases and their siblings were genetically compared. Viral loads in DBSs obtained from some of the cases were compared with those in the urine filters.

Results: Congenital CMV infection was identified in $0.31 \%(95 \% \mathrm{Cl} 0.24 \%$ to $0.39 \%$ ) of the newborns, and $30 \%$ of the cases $(20 / 66)$ had typical clinical manifestations and/or showed abnormalities in brain images at birth. Although the positive predictive value of our screening was $94 \%$, the lack of any comparison with a gold standard assay prevented calculation of the negative predictive value. Almost two-thirds of the cases had siblings, a significantly higher frequency than for uninfected newborns. Most of the cases (21/ 25) excreted CMV strains identical to those of their siblings. CMV DNA was undetectable in three out of 12 retrievable DBS specimens.

Conclusions: Implementation of an effective large-scale screening programme for congenital CMV infection is feasible. Siblings are the major risk factor for congenital CMV infection, which emphasises the need for education of mothers-to-be as well as vaccine development.

\section{ARTICLE SUMMARY}

Article focus

- It is critical to establish a strategy for screening newborns infected congenitally with cytomegalovirus (CMV), as they are at risk for the development of neurological sequelea. However, because of the insufficient sensitivity of newborn CMV screening based on dried blood spots, the feasibility of an alternative approach using urine specimens needs to be evaluated.

- Not only population-based epidemiologica studies, but also prospective molecular studies, are necessary to clarify the transmission routes of congenital CMV infection.

Key messages

- Collection of urine specimens onto filter cards placed in the diapers made large-scale screening for congenital CMV infection feasible and effective without compromising detection sensitivity.

- One out of every 300 newborns is congenitally infected with CMV, and $30 \%$ of the cases were symptomatic in the society where the CMVseroprevalence is $70 \%$, indicating that the frequency of congenital CMV infections is a medical problem of the same level as Down's syndrome.

- Our direct molecular evidence indicates that siblings are the major risk for congenital CMV infection.

Strengths and limitations of this study

- This multicentre large-scale screening study demonstrates that our urine-filter-based method is robust and reliable. However, the lack of any comparison with a gold standard assay prevented the determination of the exact frequency of false-negative results.

- Serological studies on mothers need to be combined with newborn screening to confirm whether transmission predominantly occurs via maternal primary infection. 


\section{INTRODUCTION}

Congenital cytomegalovirus (CMV) infection occurs in $0.2-2 \%$ of births in developed countries and causes significant clinical consequences not only at birth but also later as neurological sequelae, including sensorineural hearing loss (SNHL) and developmental delay. ${ }^{1}$ Our retrospective studies demonstrated that $12-15 \%$ of cases with severe SNHL and $25 \%$ of cases with developmental delay of unknown cause were associated with fetal congenital CMV infection; half of the sequelae were of late onset. ${ }^{2}{ }^{3}$ Early identification of congenital CMV infection may lead to new treatment options with antiviral agents. ${ }^{4}$ In addition, early intervention in infants with SNHL enables language development to reach a level comparable with that of audiologically normal peers. ${ }^{5}$ As many cases have late-onset SNHL, the ability of standard newborn hearing screening to detect congenital CMV-associated SNHL is limited. ${ }^{6}$ Therefore, it is important to establish newborn congenital CMV screening programmes.

Traditionally, diagnosis of congenital CMV infection has been performed by the culture of CMV from urine specimens collected within 3 weeks of birth. As the collection of liquid urine specimens is laborious, recent studies have examined the utility of dried blood spots (DBSs) for congenital CMV diagnosis. ${ }^{78}$ The arguments in support of DBS-based assay rely on the presence of infrastructure for DBS collection and their cost efficiency. However, several studies have found that DBSbased assays are of limited sensitivity. ${ }^{9}{ }^{10}$ Virus loads in blood, particularly from asymptomatic cases, are very low, ${ }^{11}{ }^{12}$ which necessitates the use of large DBS discs, efficient DNA extraction and sensitive PCR assays. ${ }^{13} 14$ To overcome the limitations associated with DBS-based assays, we developed a quantitative PCR (qPCR) assay using urine specimens collected on filter discs that can be used directly as a PCR template without additional purification or elution steps. ${ }^{11} 15$

In this study, we established a multicentre congenital CMV screening programme and tested specimens from 21272 newborns to evaluate the feasibility of our assay for a relatively large-scale screening programme, to learn the prevalence of congenital CMV infection and diseases, and to identify risk factors for congenital CMV infection in Japan.

\section{METHODS}

\section{Study design}

The collection and use of human-subject materials was approved by the Ethical Committee on Human Subjects of each participating institute. We obtained informed consent from the parents of all enrolled newborns. The screening method and study outline are illustrated in figure 1. The National Institute for Infectious Diseases (NIID) in Tokyo distributed filters with ID numbers to all study sites. The ID numbers consisted of five digit numbers, indicating $<$ area $>-<$ study site within the area $>-<$ three digits for each newborn ID at the site $>$. We collected urine on a $2 \mathrm{~cm} \times 5 \mathrm{~cm}$ piece of FTA-Elute filter

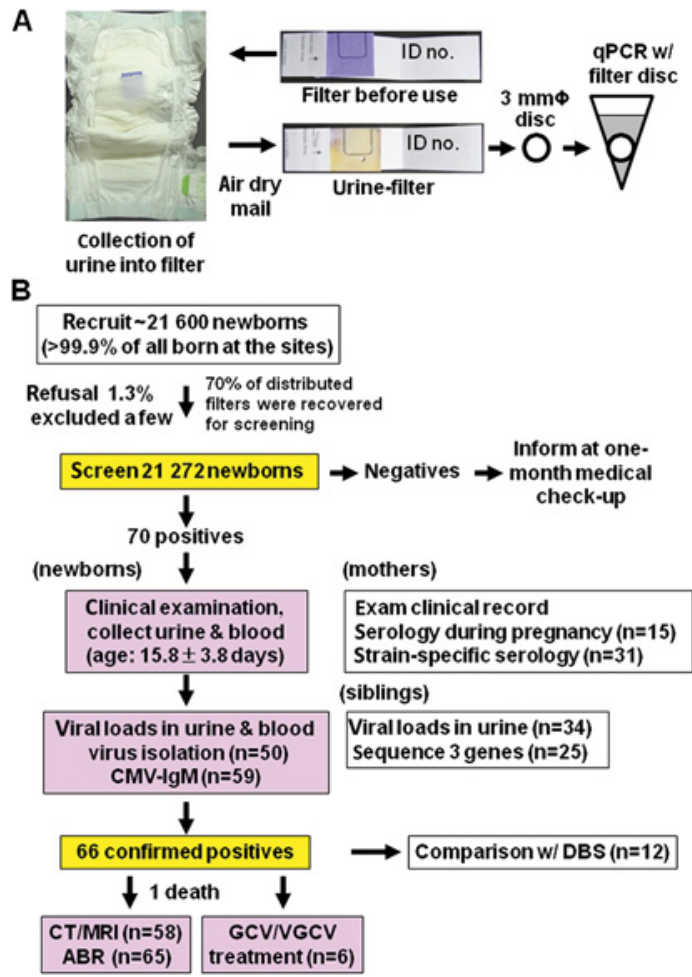

Figure 1 Screening method and study design. ABR, auditory brainstem responses; CMV, cytomegalovirus; DBS, dried blood spot; GCV, ganciclovir; VGCV, valganciclovir.

cards (Whatman) inserted into the diaper of each newborn within 4 days of birth and before discharge. After removal from the diaper, the urine filter was dried and mailed to NIID, where it was received within 1-2 days of shipment. The qPCR described in the following section was performed shortly after receipt of the specimens (mean of $1.1 \pm 1.2$ days). Approximately $70 \%$ of distributed filter cards were recovered for screening. The main reasons for the non-return of filters were (1) that they were used to demonstrate urine collection techniques to the nurses and paramedicals; (2) repeated urine collection owing to insufficient urination prior to diaper change; (3) too much stool on the filter; and (4) misplacement of the initial filter. All filters were accounted for as the NIID sent each study site an Excel file containing information regarding filter ID, date of receipt of the specimens, and test results after each qPCR run, and each study site added the results to the newborns' medical records. There were about 20 occasions on which the study sites emailed or called NIID because of a typographical error in the filter ID in the file.

When the test result was positive, physicians contacted the parents to arrange an immediate clinic visit for the collection of urine and blood specimens from the newborns to confirm congenital CMV infection. Specimens for confirmation were collected at the age of $15.8 \pm 3.8$ days (range 7-21 days). Virus isolation, virus load measurement and serology were performed using these specimens. In addition to urine specimens, dried umbilical cord specimens were obtained from 23 of the cases for further confirmation. 
We obtained separate informed consent to use DBS specimens from the cases retrospectively for our study, and requested the incorporated foundations handling DBSs for sending the specimens. DBS specimens were retrieved from 12 of the cases, 11 of which were asymptomatic, and one had SNHL at birth. Blood specimens were obtained from mothers and urine specimens from siblings.

We established 25 study sites in six geographically separate areas of Japan. The study sites serve rural, rural-urban, and urban/metro societies. More than 99.9\% of all babies born at the sites were recruited for enrolment, and $1.3 \%$ of their parents refused participation. A few infants severely ill in neonatal intensive care units (NICUs) were excluded owing to difficulties in the collection of urine. Enrolment was carried out from April 2008 to September 2010. The sites included primary obstetric clinics and municipal hospitals (type 1 sites), as well as university-associated and national hospitals (type 2 sites) that care for the general population of pregnant women as well as patients referred from type 1 clinics. Pregnant women are able to select clinics/hospitals according to their preference. Type 2 sites are generally large in scale, have a NICU and employ specialists in most clinical areas. Type 1 sites include Mori Hospital, Tomakomai City Hospital, Sapporo Tokusyukai Hospital, Nihonmatsu Hospital, Yamaguchi Hospital, Toyokawa City Hospital, Wakamiya Hospital, Palmore Hospital, Hanamizuki Ladies Clinic, Fujita Clinic, Fuchi Ladies Clinic, Miura Clinic for Women and Children, Miyamura Hospital and Takara Maternity Clinic. Type 2 sites include National Fukushima Hospital, National Centre for Child Health and Development, Iwaki Kyoritsu Hospital, Hyogo Prefectural Kobe Children's Hospital and Kariya Toyota General Hospital, and hospitals at Asahikawa Medical University, Fukushima Medical University, Tokyo University, Fujita Health University, Kobe University and Nagasaki University.

\section{qPCR for screening}

We punched a $3 \mathrm{~mm}$ diameter disc from the urine filters, washed this with $200 \mu \mathrm{l}$ of water and then transferred it into $50 \mu \mathrm{l}$ of qPCR reaction mixture in a well of a 96-well plate. Only one filter disc can be submerged into the reaction mixture after brief centrifugation $(400 \mathrm{~g}, 30 \mathrm{~s})$. The qPCR assay for the CMV UL84 fragment and thermal cycling conditions were described previously. ${ }^{2} 15$ Although it is ideal to include an internal control to ensure the absence of PCR inhibition, we did not do so in this study for the following reasons: (1) although we initially spiked irrelevant DNA/primers/probe into the qPCR reaction mixture for screening $(n=200$ urinefilters) to check the efficiency of qPCR, no cases of inhibition were observed; (2) our previous study ${ }^{15}$ demonstrated a close correlation in viral load measurements between the urine-filter-based qPCR assay and the common qPCR assay using DNA samples purified from liquid urine; and (3) to reduce cost as far as possible. To confirm positive results in the screening qPCR, DNA samples were recovered from four $3 \mathrm{~mm}$ diameter filter discs by incubation at $95^{\circ} \mathrm{C}$ for $30 \mathrm{~min}$ in $50 \mu \mathrm{l}$ of water containing $100 \mathrm{ng}$ of carrier DNA, and $5 \mu \mathrm{l}$ aliquots of the recovered DNA samples were used for qPCR. ${ }^{15}$ Simple mathematical consideration of the detection efficiencies $(15-20 \%$ in qPCR containing urine-filter vs $60 \%$ in qPCR using eluted DNA samples) and the number of used filter discs indicates that both qPCR assays detect almost equivalent amounts of CMV DNA. The cost for our test itself, including the filter paper, reagents, disposables and technical labour for the assay, was $<750$ Yen $(\sim £ 5.50, € 7$ or US\$9) per study participant.

\section{DNA preparation from specimens other than urine filters}

DNA samples were purified from liquid urine using a Viral RNA kit (Qiagen, Hilden, Germany), and from whole blood using a QIAamp DNAmini kit (Qiagen) according to the manufacturer's instructions. DNA was purified from dried umbilical cord ${ }^{2}$ and $\mathrm{DBS}^{8}$ specimens as described previously. The efficiency of DNA recovery from DBS specimens was $>90 \%{ }^{11}$

\section{Clinical and audiological evaluation}

Clinical data for newborns, including birth weight, gestational age (GA), clinical manifestations and abnormal laboratory findings, were extracted from their medical records. The mental and physical development of infected infants has been closely monitored at outpatient clinics. Audiological testing was carried out using auditory brainstem responses and/or auditory steady-state responses, and brain imaging was carried out by CT and/or magnetic resonance images. Although interpretation of mild abnormalities in brain images is sometimes difficult, our Study Group contains a specialist with long experience in paediatric neurology and brain imaging. Medical records of mothers were examined for abnormalities during pregnancy. We defined 'typical clinical manifestations' as any of microcephaly, chorioretinitis, SNHL or a combination of petechiae, hepatosplenomegaly and jaundice. Intrauterine growth restriction (IUGR) and any single, mild manifestation were discounted as 'typical clinical manifestations.' 'Symptomatic' cases were defined as those exhibiting any typical clinical manifestations and/or abnormalities in their brain images.

Prior to the initiation of screening, our Study Group prepared a tentative guideline for antiviral treatment based on the same protocol and inclusion/exclusion criteria used in the published clinical trial. ${ }^{16}$ Treatment was performed based on the criteria and informed consent.

\section{Strain analysis and serology}

Sequences of the polymorphic regions of the gN, UL144, and UL146 genes of CMV strains were determined as described previously. ${ }^{17} 18$ In contrast to European countries, only $10 \%$ of obstetric clinics/hospitals in 
Japan conduct routine CMV serology for pregnant women. In this study, two study sites performed a CMVspecific IgG test for all pregnant mothers $(n=4877)$, and some sites tested CMV-IgG of pregnant women with any risk factors $(n=524)$ at $10-20$ weeks of pregnancy. Each of those sites used one of the following additional tests; CMV-IgG avidity test for seropositives at 19 weeks of pregnancy, CMV-IgG test for seronegatives at 35-36 weeks of pregnancy, and CMV-IgG and - IgM tests for seronegatives during the middle and late terms of pregnancy. All of their babies except for refusals were enrolled for CMV screening after birth. Serology tests for CMV-specific IgG and IgM were carried out at a commercial laboratory (SRL, Tokyo, Japan) using EIA kits produced at DENKA SEIKEN Co. (Niigata, Japan). The CMV-IgM kit is based on the IgM-captured sandwich method using CMV-specific antibodies for detection. The CMV-IgG avidity test was carried out at Aisenkai Nichinan Hospital as described previously. ${ }^{19}$

\section{Statistical methods}

Statistical significance was evaluated using the $\chi^{2}$ test. The Mann-Whitney $\mathrm{U}$ test was used to analyse differences in virus loads between two groups. We used a website (http://www.measuringusability.com/wald. htm) to calculate adjusted Wald $95 \%$ CIs.

\section{RESULTS}

\section{Prevalence of congenital CMV infection}

Seventy of 21272 newborns were positive in the initial urine-filter screening, and all of these were confirmed positive by the second PCR using DNA samples recovered from the urine filters. The positive results were re-examined with urine specimens collected in liquid form. In two cases, dried umbilical cord specimens were used for the confirmation, as there was a delay in urine-filter collection owing to accommodation in an NICU in one case and a delayed clinic visit in the other case. Among the 70 cases positive in the initial screening, five urine filters yielded 9-90 CMV copies/reaction (estimated $3 \times 10^{4} \sim 3 \times 10^{5}$ copies/ml urine), which was smaller than those of the other cases ( $>200$ copies/reaction), and only one of these five cases was positive for CMV in the liquid urine specimens $\left(5.8 \times 10^{4} / \mathrm{ml}\right)$. CMV was successfully cultured from all available urine specimens obtained from the screening-positive cases $(n=50)$. In addition to the urine specimens, dried umbilical cord specimens from the cases $(n=23)$ were used for further confirmation. In all, congenital CMV infection was confirmed in a total of 66 newborns $(0.31 \%$; $95 \%$ CI 0.24 to $0.39 \%$ ) (table 1$)$. There were no statistically significant differences in the prevalence of congenital CMV infection among the six geographical areas, although life styles ranged from metropolitan to traditional (data not shown).

Type 2 sites have more patients referred by primary clinics/hospitals (type 1 sites), and some type 2 sites enrolled newborns who required NICU care. Thus, there was a difference between the type 1 and type 2 sites in the proportion of newborns with a birth weight $<2500 \mathrm{~g}$ (5.1\% vs $13.5 \%)$ but not in that of newborns small for GA (SGA) $(5.1 \%$ vs $5.9 \%)$. The prevalence of congenital CMV infections differed approximately twofold between the two types of study sites (table 1).

\section{Characteristics of CMV-infected newborns}

Typical clinical manifestations, defined as any of microcephaly, chorioretinitis, SNHL or a combination of petechiae, hepatosplenomegaly and jaundice, were observed in $22.7 \%(15 / 66)$ of the infected newborns (table 1). SNHL was observed in 53\% (8/15) of those cases. Abnormalities in brain images, including intracranial calcifications, ventricular dilation and abnormal lesions, were identified in 10 out of 58 cases. The proportion of cases with typical clinical manifestations and/or with the abnormalities in brain images was $30.3 \%(20 / 66)$. As we did not include cases with a single, mild clinical manifestation and because brain imaging was not available for eight cases, the proportion of socalled 'symptomatic' cases was likely to have been underestimated. Cases identified at type 2 sites had more frequent clinical manifestations and abnormalities.

Virus loads in whole blood, but not urine, from the symptomatic cases were statistically higher than those from asymptomatic cases (supplementary figure). Virus loads in the urine were 16- to 22000 -fold higher than those in the blood.

Table 1 Comparison of prevalence and clinical outcomes between types of study sites

\begin{tabular}{|c|c|c|c|}
\hline & $\begin{array}{l}\text { All sites } \\
(n=25)\end{array}$ & $\begin{array}{l}\text { Type } 1 \text { sites* } \\
(n=14)\end{array}$ & $\begin{array}{l}\text { Type } 2 \text { sites } † \\
(n=11)\end{array}$ \\
\hline No of screened newborns & 21272 & 14642 & 6630 \\
\hline No of congenitally infected cases & 66 & 35 & 31 \\
\hline Prevalence of congenital infection, $\%(95 \% \mathrm{Cl})$ & $0.31(0.24$ to 0.40$)$ & $0.24(0.16$ to 0.32$)$ & $0.47(0.30$ to 0.63$)$ \\
\hline \multicolumn{4}{|l|}{ Abnormalities identified within 3 months after birth } \\
\hline A: typical clinical manifestations at birth & $22.7 \%(n=15)$ & $8.6 \%(n=3)$ & $38.7 \%(n=12)$ \\
\hline B: abnormalities in brain images (CT/MRI) & $15.2 \%(n=10)$ & $11.4 \%(n=4)$ & $19.4 \%(n=6)$ \\
\hline A and/or B & $30.3 \%(n=20)$ & $17.1 \%(n=6)$ & $45.2 \%(n=14)$ \\
\hline Treated with antiviral drugs & $9.1 \%(n=6)$ & $0.0 \%$ & $22.6 \%(n=6)$ \\
\hline Detectable cytomegalovirus-IgM in blood of the cases & $50.8 \%(30 / 59)$ & $51.6 \%(16 / 31)$ & $50 \%(14 / 28)$ \\
\hline
\end{tabular}


Of the 66 confirmed cases, six newborns, who exhibited clinically obvious symptoms, including IUGR, petechiae, hepatosplenomegaly and jaundice, accompanied by the following abnormalities, SNHL $(n=4)$, chorioretinitis $(n=1)$ and abnormal brain images $(n=3)$, were treated with ganciclovir (GCV) and/or valganciclovir (VGCV) immediately after diagnosis. In spite of the intensive treatment, one infant died. In addition, one child with late-onset SNHL was treated with VGCV at 5 months after birth, and an improvement in SNHL was observed. $^{20}$

\section{Newborns with congenital CMV frequently have siblings}

The general characteristics of the newborns enrolled in this study are summarised in table 2. It is likely that the uninfected participants represent our average national population, since the 2009 National Vital Statistics reported by the Ministry of Health, Labour, and Welfare in Japan indicates that the average mothers' age, the average birth weight and the proportion of newborns with a birth weight of $<2500 \mathrm{~g}$ are 31.0 years, $3000 \mathrm{~g}$ and $7.7 \%$, respectively, similar to the values observed in our study population.

Mothers' age and GA of the infected newborns were similar to those of the uninfected newborns. However, the average birth weight of the infected newborns was slightly less than that of the uninfected newborns. The differences in the proportion of newborns with a birth weight of $<2500 \mathrm{~g}$ and that of SGA were statistically significant, with the differences being more significant in symptomatic cases.

One of the most important findings of this study is that infected newborns were more likely to have siblings $(63.6 \%$ vs $48.8 \%, \mathrm{p}<0.02)$. Among the 42 cases with siblings, 34 had one sibling, seven had two, and one had three.

\section{Comparison of CMV strains between cases and siblings}

We collected urine specimens from the siblings of 34 infected newborns. Twenty-five of the siblings' specimens contained sufficient CMV DNA for PCR amplification of the polymorphic regions of the gN, UL144 and UL146 genes. Eighty-four per cent $(21 / 25)$ of the pairs had identical DNA sequences in these genes (table 3 ). The mother of one of the four newborns for whom the sibling strain was not identical is a nurse working in the department of internal medicine. There was no significant linkage of particular genotype(s) with clinical outcomes. In addition to the 21 pairs, three additional pairs, symptomatic cases identified at hospitals outside the study sites and their siblings, were infected with the same strains (data not shown).

\section{Technical issues}

The first issue is the fact that the detection of CMVspecific IgM is insensitive for newborn congenital CMV screening, since CMV-specific IgM was detectable in only half $(30 / 59)$ of the congenital CMV cases (table 1 ). The second issue is the choice of specimens for screening. Viral load estimates obtained from urinefilters were in good agreement with those determined from liquid urine specimens (figure 2). Viral load estimates from the DBS specimens from the cases $(n=12)$ also showed fair agreement with those determined using blood specimens. Importantly, the total CMV DNA load in three or four discs of DBS specimens was far less than that in a single urine-filter disc, and three out of 12 DBS specimens were negative in the PCR assay, the sensitivity of which was previously demonstrated to be $>90 \%$. Finally, adverse events associated with filter screening were observed in $0.15 \%$ of the study population. These were mostly minor skin irritations (ie, redness of skin), but four babies, including two premature newborns with a body weight $<1000 \mathrm{~g}$, experienced severe perianal skin erosions. Mistakes such as placing the filter directly onto the skin rather than underneath the top layer of the diaper and leaving the diaper unchanged for an excessive period were the probable cause.

\begin{tabular}{|c|c|c|c|c|}
\hline & $\begin{array}{l}\text { Uninfected } \\
\text { screened newborns } \\
n=18330^{\star}\end{array}$ & $\begin{array}{l}\text { Infected cases } \\
n=66\end{array}$ & p Value & $\begin{array}{l}\text { Infected cases with } \\
\text { abnormalities at birth } \dagger \\
n=20\end{array}$ \\
\hline Mean \pm SD of mothers' age (years old) & $31.2 \pm 4.8$ & $29.7 \pm 5.9$ & NS & $30.6 \pm 5.0$ \\
\hline Mean $\pm S D$ of gestation age & $39 w 1.9 d \pm 1 w 2.1 d$ & $38 w 3.9 d \pm 2 w 6.1 d$ & NS & $38 w 3.7 d \pm 2 w 5.2 d$ \\
\hline Mean $\pm S D$ of birth weight $(g)$ & $3002.9 \pm 385.2$ & $2756.4 \pm 463.7$ & NS & $2589.5 \pm 515.0$ \\
\hline $\begin{array}{l}\text { Proportion of low birth weight } \\
(<2500 \mathrm{~g}), \%(95 \% \mathrm{Cl})\end{array}$ & $7.3(6.9$ to 7.7$)$ & 22.7 (14.1 to 34.3$)$ & $<0.001$ & 40 (21.8 to 61.4$)$ \\
\hline $\begin{array}{l}\text { Proportion of those small for gestational } \\
\text { age } \neq, \%(95 \% \mathrm{Cl})\end{array}$ & 5.3 (5.0 to 5.6$)$ & 18.2 (10.6 to 29.3$)$ & $<0.001$ & 30 (14.3 to 52.1$)$ \\
\hline $\begin{array}{l}\text { Proportion of the cases with elder } \\
\text { sibling(s), \% }(95 \% \mathrm{Cl})\end{array}$ & 48.8 (48.1 to 49.5$)$ & 63.6 (51.6 to 74.2$)$ & $<0.02$ & 60 (38.6 to 78.2$)$ \\
\hline
\end{tabular}




\begin{tabular}{|c|c|c|c|c|c|c|c|}
\hline \multirow[b]{2}{*}{ No } & \multicolumn{3}{|c|}{ Genotypes of case } & \multirow{2}{*}{$\begin{array}{l}\text { Sequences between } \\
\text { case and sibling(s) }\end{array}$} & \multicolumn{3}{|c|}{ Genotypes of sibling(s) } \\
\hline & gN & UL144 & UL146 & & gN & UL144 & UL146 \\
\hline 1 & 1 & $A$ & 7 & Identical & 1 & $A$ & 7 \\
\hline 2 & 1 & $A$ & 11 & Identical & 1 & $A$ & 11 \\
\hline 3 & 1 & B & 12 & Identical & 1 & $\mathrm{~B}$ & 12 \\
\hline 4 & 1 & $\mathrm{C}$ & 12 & Identical & 1 & C & 12 \\
\hline 5 & 2 & A & 13 & Identical & 2 & $A$ & 13 \\
\hline 6 & 2 & B & 5 & Identical & 2 & B & NA \\
\hline 7 & 2 & B & NA & Identical & 2 & NA & NA \\
\hline 8 & $3 a$ & $A$ & 9 & Identical & $3 a$ & $A$ & 9 \\
\hline 9 & $3 a$ & $A$ & 9 & Identical & $3 a$ & A & 9 \\
\hline 10 & $3 a$ & $\mathrm{C}$ & 12 & Identical & $3 a$ & C & 12 \\
\hline 11 & $3 b$ & $\mathrm{C}$ & 11 & Identical & $3 b$ & C & 11 \\
\hline 12 & $4 a$ & $A$ & 8 & Identical & $4 a$ & A & 8 \\
\hline 13 & $4 a$ & $A$ & 11 & Identical & $4 a$ & A & NA \\
\hline 14 & $4 a$ & $A$ & 11 & Identical & $4 a$ & $A$ & 11 \\
\hline 15 & $4 a$ & $B$ & 1 & Identical & $4 a$ & $B$ & 1 \\
\hline 16 & $4 a$ & C & 12 & Identical & $4 a$ & C & 12 \\
\hline 17 & $4 b$ & $A$ & 9 & Identical & $4 b$ & $A$ & 9 \\
\hline 18 & $4 b$ & $B$ & 1 & Identical & $4 b$ & $B$ & 1 \\
\hline 19 & $4 b$ & B & 12 & Identical & $4 b$ & B & 12 \\
\hline 20 & $4 b$ & C & 11 & Identical & $4 b$ & C & 11 \\
\hline 21 & $4 c$ & $B$ & 9 & Identical & $4 c$ & $B$ & 9 \\
\hline 22 & 1 & B & 12 & Different & 1 & A & 1 \\
\hline 23 & 2 & B & 6 & Different & $3 a$ & $A$ & 9 \\
\hline 24 & $3 a$ & $A$ & 1 & Different & 2 & B & 12 \\
\hline 25 & $4 c$ & A & 7 & Different & $3 b$ & B & 13 \\
\hline 26 & 2 & $B$ & 9 & Low copy & & & \\
\hline 27 & $3 a$ & B & 6 & Low copy & & & \\
\hline 28 & $3 a$ & $\mathrm{C}$ & 12 & Low copy & & & \\
\hline 29 & $3 a$ & NA & 9 & Low copy & & & \\
\hline 30 & $3 a$ & A & 7 & Low copy & & & \\
\hline 31 & $3 b$ & $B$ & 5 & Low copy & & & \\
\hline 32 & $4 a$ & $A$ & 8 & Low copy & & & \\
\hline 33 & $4 c$ & $B$ & 11 & Low copy & & & \\
\hline 34 & NA & NA & NA & Low copy & & & \\
\hline
\end{tabular}

\section{DISCUSSION}

There were three major outcomes of our study. First, we demonstrated that the collection of urine specimens in diapers followed by high-throughput qPCR is feasible for large-scale screening for congenital CMV. Second, we provided additional evidence that virus loads in the blood of symptomatic cases are higher than those in cases who are asymptomatic at birth ${ }^{12} 21$ and that virus loads in the urine are several orders of magnitude higher than those in blood, ${ }^{11}$ thereby enabling urine-based screening to detect more congenital CMV cases. Finally, we found that (1) two-thirds of congenital CMV cases had siblings, (2) most of the siblings were actively excreting large amounts of CMV in their urine, and (3) most of the sibling pairs were excreting identical strains of CMV. The prevalence of congenital CMV infection, one out of every 300 newborns, and the presence of clinical manifestations in $30 \%$ of the infected infants indicate that congenital CMV infection is a serious medical problem at a level similar to that of Down's syndrome, as demonstrated in other studies. ${ }^{1} 22$
We found that the urine-filter collection was not a burden, either on the newborns or on clinic/hospital staffs, once they understood the work flow, although it required extra labour in comparison with DBS-based screening. Since most babies in Japan are delivered at clinics/hospitals and stay for 5-6 days, we cannot predict whether our approach is applicable for babies delivered at home. However, in one of our studies, we provided 30 mothers with filters and an illustration showing how to collect urine from their 1 2-year-old children at home, and found that most mothers were able to send the urine filters back without any trouble, suggesting that home collection is possible. The use of an auto-puncher and a bar-code system may increase the assay throughput and reliability, although we did not try this, owing to limited resources.

We should note that one of the limitations of our study is the lack of any comparison with a gold-standard screening method, which made it impossible to identify false-negative cases, although the higher viral loads in urine are expected to increase the sensitivity of our 


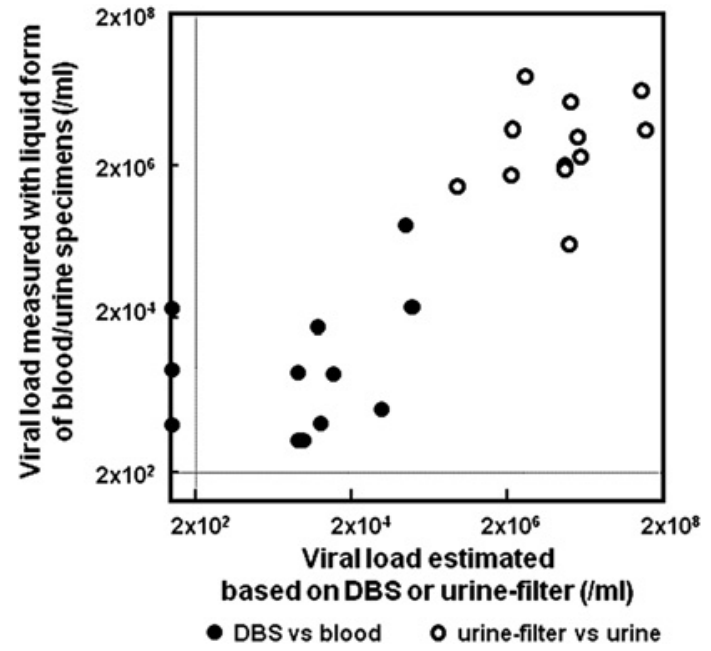

Figure 2 Comparison of viral loads in $1 \mathrm{ml}$ of whole blood or urine that were estimated based on dried-blood-spot- (DBS)- or urine-filter-based PCR assay ( $\mathrm{x}$-axis) with those that were measured by qPCR using a liquid form of blood or urine specimens (y-axis). Closed and open circles indicate the comparison between DBS-based estimates and measurements of the whole-blood specimens and between urine-filter-based estimates and measurements of urine specimens, respectively. The detection limit of the assays (indicated with broken lines) was 200 copies $/ \mathrm{ml}$.

screening method. The positive predictive value for congenital infection in our screening was $94 \%$; in other words, there were $6 \%$ false-positive rates, which emphasises the importance of confirmation using additional materials. We think that this issue is not unique to our study. The major challenge remaining is how to obtain additional materials as early as possible, since postnatal infection via breast milk may interfere with the PCRbased diagnosis of congenital infection if the materials are collected after 2 weeks postpartum. ${ }^{23}$ Although viral loads in congenitally infected infants are usually larger than those in postnatally infected infants, virus isolation is advised. Fortunately, in Japan we can use dried umbilical cords stored at home for further confirmation. ${ }^{2}{ }^{3} \mathrm{~A}$ period (probably $3-5$ days) from the collection of urine filters to their shipping out can be reduced, since not all study sites sent the filters in a timely manner. We do not have a clear explanation for the causes of the four falsepositive results. At least one of them was collected at the same site on the same day as a confirmed congenital case, which suggests the possibility of contamination.

In this study, typical clinical manifestations and/or the brain image abnormalities were observed in $30 \%$ of the cases. Although the proportion of cases with clinical manifestations seems to be relatively higher than those reported previously, some studies have reported figures similar to ours. ${ }^{24}$ Importantly, the disease definitions applied here were similar to those used in most studies. One of the factors that may have affected the epidemiological outcomes is the selection of study sites. The prevalence at the type 1 sites may be underestimated, since mothers with any pregnancy-associated risk factors, such as IUGR, tend to choose type 2 hospitals that have an NICU, well-equipped facilities and specialists. In addition, physicians at type 1 sites prefer to refer those mothers to type 2 hospitals. Actually, the proportion of newborns with a birth weight $<2500 \mathrm{~g}$ at the type 1 sites was smaller than that at the type 2 sites. In other words, the prevalence at type 2 sites may have been overestimated. Although, ideally, we should know the proportions of babies born at type 1 and type 2 clinics/ hospitals in our country, such statistics are not available. Therefore, the true prevalence of CMV infection and disease in Japan is likely to be somewhere between those of the type 1 and type 2 sites.

Our finding that congenital CMV cases were more likely to have siblings and that case/siblings CMV strains are often identical is important. Having young children and recent onset of sexual activities are epidemiologically major risks for congenital CMV. ${ }^{22} 2526$ A population-based study among preadolescents in the USA demonstrated the importance of family transmission rather than childcare-centre attendance. ${ }^{27}$ However, demonstration of a relationship between CMV strains from sources and cases has been limited to comparison by restriction-enzyme fragment length polymorphism analysis. $^{28}{ }^{29}$ Since CMV genome sequences are well conserved, we employed a more robust method based on the DNA sequences from three polymorphic genes. This enabled us to demonstrate directly that siblings play a major role in congenital CMV infections. Related to this, we have found that $27 \%(29 / 108)$ of $1-5$-year-old healthy children excrete $>10000$ copies $/ \mathrm{ml}$ of $\mathrm{CMV}$ in their urine (NI, unpublished data).

An important question is whether maternal-fetal transmission occurs during maternal primary infection, during reactivation or after reinfection with a different strain of the virus. A recent meta-analysis demonstrated that average rates of primary and recurrent infections were $32 \%$ and $1.4 \%$, respectively. ${ }^{24}$ Strain-specific serology showed that two-thirds of congenital CMV infections in seropositive women in Alabama were caused by reinfection, ${ }^{30}$ and that reinfections with different strains are common in healthy women in the USA and Brazil. ${ }^{31}{ }^{32}$ CMV seroprevalence in Japan is estimated to be $71.2 \%$, ranging geographically from $69 \%$ to $76 \%,{ }^{33}$ which is lower than those in Alabama and Brazil, and similar to those $(40-80 \%)$ in European countries. ${ }^{34}$ A limitation of this study is that serological data were available only for 15 of the 66 mothers of the congenital cases, since CMV serology during pregnancy is not common practice in Japan. Among the 15 mothers, five seroconverted during their pregnancies, five had CMV-specific IgM (at least three of them had a low CMV-IgG avidity index) during their pregnancies, and five were already seropositive at the early stage of pregnancy. Based on our preliminary strain-specific serology, it is unlikely that reinfection is the major route of infection in Japan (Ikuta, Suzutani, et al unpublished). 
Thus, we think that at least half of the congenital CMV infections we identified most likely occurred during maternal primary infection.

Our study suggests that young children in a family are the major risk factor for transmission to their pregnant mothers. A significant proportion of pregnant mothers may be constantly exposed to CMV at home since, as described above, at least a quarter of young children excrete $0.5-11$ of urine containing $>10000$ copies $/ \mathrm{ml}$ of CMV daily, often for $>2$ years. Because of this, development of a vaccine to prevent CMV must become a national and international priority. A recent clinical trial using $\mathrm{gB}$ subunit vaccine provided a promising direction. ${ }^{35}$ In the mean time, serological screening coupled with educational counselling of seronegative mothers-to-be can decrease seroconversion. ${ }^{36} 37$

Our study clearly demonstrates the feasibility of implementing large-scale and effective screening programmes that will enable strategies for timely clinical intervention and treatment that minimise the damage caused by congenital CMV-associated sequelae.

\section{Author affiliations \\ ${ }^{1}$ Department of Paediatrics, Asahikawa Medical University, Hokkaido, Japan ${ }^{2}$ Department of Virology I, National Institute of Infectious Diseases, Tokyo, Japan \\ ${ }^{3}$ Department of Paediatrics, Kyorin University, Tokyo, Japan \\ ${ }^{4}$ Department of Paediatrics, Nagasaki University, Nagasaki, Japan \\ ${ }^{5}$ Department of Obstetrics and Gynecology, Fukushima Medical University, \\ Fukushima, Japan \\ ${ }^{6}$ Department of Maternal and Perinatal Services, National Centre for Child Health and Development, Tokyo, Japan \\ ${ }^{7}$ Department of Obstetrics and Gynecology, Kobe University Graduate School of Medicine, Hyogo, Japan \\ ${ }^{8}$ Department of Pediatrics, Fujita Health University, Aichi, Japan \\ ${ }^{9}$ Department of Microbiology, Fukushima Medical University, Fukushima, Japan}

Acknowledgements We dedicate this article to K Fujieda, who originally administrated the study group before passing away in March 2010. We would like to thank our medical and nursing colleagues as well as the newborns and their parents who agreed to take part in this study. We also thank $P$ Pellett (Wayne State University, Detroit, Michigan) and T Kawana (Teikyo University), for their intellectual input, and $M$ Tsuda and $Y$ Fukui, for their technical assistance with PCR screening and virus isolation. We would like to thank the following members of the Japanese Congenital Cytomegalovirus Study Group members for their contributions: T Nagamori (Asahikawa Medical University), H Asanuma (Tomakomai City Hospital), Y Mori (Mori Hospital), M Ohsima (Sapporo Tokusyukai Hospital), T Imamura, K Ikuta, A Sato, K Fujimori (Fukushima Medical University), M Owada (Nihonmatsu Hospital), R Suzuki (National Fukushima Hospital), T Honda (Iwaki Kyoritsu Hospital), K Goishi, Y Mizuno (Tokyo University), S Yamaguchi (Yamaguchi Hospital), K Tsukamoto, S Fujiwara, H Nakamura, T Kubo, H Taiji, S Harada (National Centre for Child Health and Development), S Yamada (National Institute of Infectious Diseases), T Ohishi (Saitama Children's Medical Centre), M Morizane, A Sonoyama, I Morioka (Kobe University Graduate School of Medicine), T Funakoshi (Hyogo Prefectural Kobe Children's Hospital), M Ohashi (Wakamiya Hospital), K Shimogaki (Palmore Hospital), H Nakai, K Tanaka, S Go (Fujita Health University), M Moriuchi, H Masuzaki (Nagasaki University), T Hamasaki (Hanamizuki Ladies Clinic), A Fujita (Fujita Clinic), T Fuchi (Fuchi Ladies Clinic), K Miura (Miura Clinic for Women and Children), Y Miyamura (Miyamura Hospital), M Morisaki (Takara Maternity Clinic), T Minematsu (Aisenkai Nichinan Hospital). All the listed individuals are part of the Study Group and have not received any compensation.

Funding This work was supported by Grants for the Research on Child Development and Diseases (H20-Kodomo-007; H23-Jisedai-Ippan-001) from the Ministry of Health, Labour and Welfare, Japan.
Patient consent Obtained from the parents.

Ethics approval Ethics approval was provided by the Ethical Committee on Human Subjects of each participating institute.

Contributors SK, NI and K Fujieda (deceased) designed the study. SK and K Fujieda (deceased) coordinated operations at the study sites. SK and NI had full access to all of the data in this study and take responsibility for the integrity of the data and accuracy of the data analysis. SK, AO, HM, KA, YI, HY and TY enrolled newborns and acquired medical data. NI conducted all initial screening, and TS performed serological tests. NI drafted the manuscripts, and all others provided important intellectual input for revision of the manuscript.

Provenance and peer review Not commissioned; externally peer reviewed.

Data sharing statement Technical details of the screening assay and some of the raw dataset for the tables presented in the manuscript are available from the corresponding author at ninoue@nih.go.jp. Consent for data sharing was not obtained from the parent(s), but the presented data are anonymised, and the risk of identification is low.

\section{REFERENCES}

1. Pass RF. Cytomegalovirus. In: Knipe DM, Howley PM, eds. Fields Virology. Philadelphia: Lippincott Williams \& Wilkins, 2001:2675-705

2. Ogawa $\mathrm{H}$, Suzutani T, Baba Y, et al. Etiology of severe sensorineural hearing loss in children: independent impact of congenital cytomegalovirus infection and GJB2 mutations. J Infect Dis 2007;195:782-8.

3. Koyano S, Inoue N, Nagamori T, et al. Dried umbilical cords in the retrospective diagnosis of congenital cytomegalovirus infection as a cause of developmental delays. Clin Infect Dis 2009;48:e93-5.

4. Nassetta L, Kimberlin D, Whitley R. Treatment of congenital cytomegalovirus infection: implications for future therapeutic strategies. J Antimicrob Chemother 2009;63:862-7.

5. Yoshinaga-Itano $\mathrm{C}$. Early intervention after universal neonatal hearing screening: impact on outcomes. Ment Retard Dev Disabil Res Rev 2003:9:252-66

6. Korver AM, de Vries JJ, Konings S, et al. DECIBEL study: Congenital cytomegalovirus infection in young children with permanent bilateral hearing impairment in The Netherlands. J Clin Virol 2009;46(Suppl 4): S27-31.

7. Barbi M, Binda S, Caroppo S, et al. Multicity Italian study of congenital cytomegalovirus infection. Pediatr Infect Dis J 2006;25:156-9.

8. Scanga L, Chaing S, Powell C, et al. Diagnosis of human congenital cytomegalovirus infection by amplification of viral DNA from dried blood spots on perinatal cards. J Mol Diagn 2006;8:240-5.

9. Boppana SB, Ross SA, Novak Z, et al; National Institute on Deafness and Other Communication Disorders CMV and Hearing Multicenter Screening (CHIMES) Study. Dried blood spot real-time polymerase chain reaction assays to screen newborns for congenital cytomegalovirus infection. JAMA 2010;303:1375-82.

10. Barbi M, MacKay WG, Binda S, et al. External quality assessment of cytomegalovirus DNA detection on dried blood spots. BMC Microbiol 2008;8:2.

11. Inoue N, Koyano S. Evaluation of screening tests for congenital cytomegalovirus infection. Pediatr Infect Dis J 2008;27:182-4.

12. Lanari M, Lazzarotto $\mathrm{T}$, Venturi $\mathrm{V}$, et al. Neonatal cytomegalovirus blood load and risk of sequelae in symptomatic and asymptomatic congenitally infected newborns. Pediatrics 2006;117:e76-83.

13. de Vries JJ, Claas EC, Kroes AC, et al. Evaluation of DNA extraction methods for dried blood spots in the diagnosis of congenital cytomegalovirus infection. J Clin Virol 2009;46(Suppl 4):S37-42.

14. Gohring K, Dietz K, Hartleif $S$, et al. Influence of different extraction methods and PCR techniques on the sensitivity of HCMV-DNA detection in dried blood spot (DBS) filter cards. J Clin Virol 2010;48:278-81.

15. Nozawa N, Koyano S, Yamamoto Y, et al. Real-time PCR assay using specimens on filter disks as a template for detection of cytomegalovirus in urine. J Clin Microbiol 2007;45:1305-7.

16. Kimberlin DW, Lin CY, Sanchez PJ, et al. Effect of ganciclovir therapy on hearing in symptomatic congenital cytomegalovirus disease involving the central nervous system: a randomized, controlled trial. $J$ Pediatr 2003;143:16-25.

17. Yan H, Koyano S, Inami Y, et al. Genetic linkage among human cytomegalovirus glycoprotein $\mathrm{N}(\mathrm{gN})$ and $\mathrm{gO}$ genes, with evidence for recombination from congenitally and post-natally infected Japanese infants. J Gen Virol 2008;89:2275-9.

18. Bradley AJ, Kovacs IJ, Gatherer D, et al. Genotypic analysis of two hypervariable human cytomegalovirus genes. J Med Virol 2008;80:1615-23

19. Tagawa M, Minematsu T, Masuzaki H, et al. Seroepidemiological survey of cytomegalovirus infection among pregnant women in Nagasaki, Japan. Pediatr Int 2010;52:459-62. 
20. Imamura $\mathrm{T}$, Suzutani $\mathrm{T}$, Ogawa $\mathrm{H}$, et al. Oral valganciclovir treatment for congenital cytomegalovirus infection. Pediatr Int 2011;53:249-52.

21. Boppana SB, Fowler KB, Pass RF, et al. Congenital cytomegalovirus infection: association between virus burden in infancy and hearing loss. J Pediatr 2005;146:817-23.

22. Fowler KB, Pass RF. Risk factors for congenital cytomegalovirus infection in the offspring of young women: exposure to young children and recent onset of sexual activity. Pediatrics 2006;118:e286-92.

23. Hamprecht K, Maschmann J, Vochem M, et al. Epidemiology of transmission of cytomegalovirus from mother to preterm infant by breastfeeding. Lancet 2001;357:513-18.

24. Kenneson A, Cannon MJ. Review and meta-analysis of the epidemiology of congenital cytomegalovirus (CMV) infection. Rev Med Virol 2007;17:253-76.

25. Taber LH, Frank AL, Yow MD, et al. Acquisition of cytomegaloviral infections in families with young children: a serological study. $J$ Infect Dis 1985;151:948-52.

26. Yow MD, Williamson DW, Leeds LJ, et al. Epidemiologic characteristics of cytomegalovirus infection in mothers and their infants. Am J Obstet Gynecol 1988:158:1189-95.

27. Staras SA, Flanders WD, Dollard SC, et al. Cytomegalovirus seroprevalence and childhood sources of infection: a populationbased study among pre-adolescents in the United States. J Clin Virol 2008;43:266-71.

28. Pass RF, Little EA, Stagno S, et al. Young children as a probable source of maternal and congenital cytomegalovirus infection. $N$ Engl $J$ Med 1987;316:1366-70.
29. Peckham CS, Garrett AJ, Chin KS, et al. Restriction enzyme analysis of cytomegalovirus DNA to study transmission of infection. J Clin Pathol 1986;39:318-24.

30. Boppana SB, Rivera LB, Fowler KB, et al. Intrauterine transmission of cytomegalovirus to infants of women with preconceptional immunity. N Engl J Med 2001;344:1366-71.

31. Ross SA, Novak Z, Fowler KB, et al. Cytomegalovirus blood viral load and hearing loss in young children with congenital infection. Pediatr Infect Dis J 2009;28:588-92.

32. Yamamoto AY, Mussi-Pinhata MM, Boppana SB, et al. Human cytomegalovirus reinfection is associated with intrauterine transmission in a highly cytomegalovirus-immune maternal population. Am J Obstet Gynecol 2010;202:297.e1-8.

33. Azuma $\mathrm{H}$, Takanashi $\mathrm{M}$, Kohsaki $\mathrm{M}$, et al. Cytomegalovirus seropositivity in pregnant women in Japan during 1996-2009. J Jpn Soc Perin Neon Med 2011;46:1273-9.

34. Ludwig A, Hengel $\mathrm{H}$. Epidemiological impact and disease burden of congenital cytomegalovirus infection in Europe. Euro Surveill 2009; 14:26-32.

35. Pass RF, Zhang C, Evans A, et al. Vaccine prevention of maternal cytomegalovirus infection. N Engl J Med 2009;360:1191-9.

36. Picone O, Vauloup-Fellous C, Cordier AG, et al. A 2-year study on cytomegalovirus infection during pregnancy in a French hospital. BJOG 2009;116:818-23.

37. Adler SP, Finney JW, Manganello AM, et al. Prevention of child-tomother transmission of cytomegalovirus among pregnant women. J Pediatr 2004;145:485-91. 